Advances in Complex Systems

(C) World Scientific Publishing Company

\title{
MULTI-PARTICLE STRUCTURES IN NON-SEQUENTIALLY REORGANIZED HARD SPHERE DEPOSITS
}

\author{
LUIS A PUGNALONI \\ Instituto de Fisica de Liquidos y Sistemas Biologicos, \\ UNLP-CONICET, Casilla de correo 565, 1900 La Plata, Argentina \\ G C BARKER \\ Institute of Food Research \\ Norwich Research Park, Colney, \\ Norwich NR4 $7 U A$, UK \\ ANITA MEHTA \\ $S N$ Bose National Centre for Basic Sciences \\ Block JD Sector III Salt Lake, \\ Calcutta 700 098, India \\ Received (received date) \\ Revised (revised date)
}

\begin{abstract}
We have examined extended structures, bridges and arches, in computer generated, nonsequentially stabilized, hard sphere deposits. The bridges and arches have well defined distributions of sizes and shapes. The distribution functions reflect the contraints associated with hard particle packing and the details of the restructuring process. A subpopulation of string-like bridges has been identified. Bridges are fundamental microstructural elements in real granular systems and their sizes and shapes dominate considerations of structural properties and flow instabilities such as jamming.
\end{abstract}

\section{Introduction}

There has always been a fascination, amongst physicists, with the structures and configurations that exist within disordered packings of hard particles (see for example, (1]). One interest stems from the fundamental, frustrated, geometries that exist within sphere packings, e.g. [2], [3]; another comes from the parallels between random packings and the structures of real disordered materials like liquids, glasses and granular solids, 沟, 河]. In particular it is clear that the mechanical and transport properties of mesoscale disordered materials, like powders and deposits, are strongly dependent on the relative positions and connectivity of the constituent particles. A striking example of this interplay follows when several particles combine to form an 'arch' or 'bridge' near to the outlet of a gravity flow container and cause the flow to stop. This blocking phenomenon has an enormous impact on a wide 
range of technological and industrial processes; there have been many attempts to quantify the effect and to optimize operational parameters like the outlet size and the internal granular flow pattern, e.g. [6]. However there is little information on the statistical details of the particulate configurations that are the underlying cause of the blocking.

In two dimensions arches and bridges can be observed throughout dense, random, packings of hard disks, e.g. [7], and they appear to be ubiquitous elements of stable granular structures. In a recent report To et al., [8], described experiments in which a jamming arch of monosized disks was repeatedly formed, in gravitational flow, across the outlet of a conical, two-dimensional, hopper. These experiments indicated that the jamming arches had configurations that were similar to those of self-avoiding random walks. This statistical appreciation was used to obtain predictions of crucial macroscopic parameters like the jamming probability.

Below we give some details of bridge structures formed in models of hard sphere deposits and explore the role of bridges in three-dimensional disordered packings which are stable under gravity. We do not find 'diffusive' bridge configurations but we have identified a special, chain-like, subpopulation of bridges.

In a stable packing of hard particles each particle rests on three others in such a way that its weight vector passes through the triangle formed from the three contact points (We do not consider situations involving non-point contacts). A bridge is a configuration in which the three point stability conditions of two or more particles are linked i.e. there are mutual stabilizations. In a simple example, with two particles A and B, particle A would rest on particle B and two other particles whereas particle B would rest on particle A and two further particles. Neither particle $\mathrm{A}$ or $\mathrm{B}$ could rest, i.e. be part of the stable structure, without the other. Bridge configurations, therefore, are the result of non-sequential stabilizations; they cannot be formed by the sequential placement of individual particles. In practice almost all processing operations involving granular materials such as pouring and shaking etc., are non-sequential processes. Two examples of two particle bridges are shown in figure 1. Each of these configurations is part of a large, dense, packing of spheres; all those spheres not involved in the bridge have been deleted so that it is clearly visible. The configuration on the left uses only three particles as the base of the bridge whereas that on the right uses four (base particles whilst ensuring the stability of the configuration don't, themselves, involve mutual stabilizations with other particles in the bridge structure). The details of the bridge configurations, in terms of sizes and shapes, are a manifestation of the volume and angular constraints that exist in dense hard particle assemblies. In turn these structures reflect the nature of the processing operations that precede the formation of a stable packing. In this respect bridges can be seen as part of the 'memory' of a granular system. 

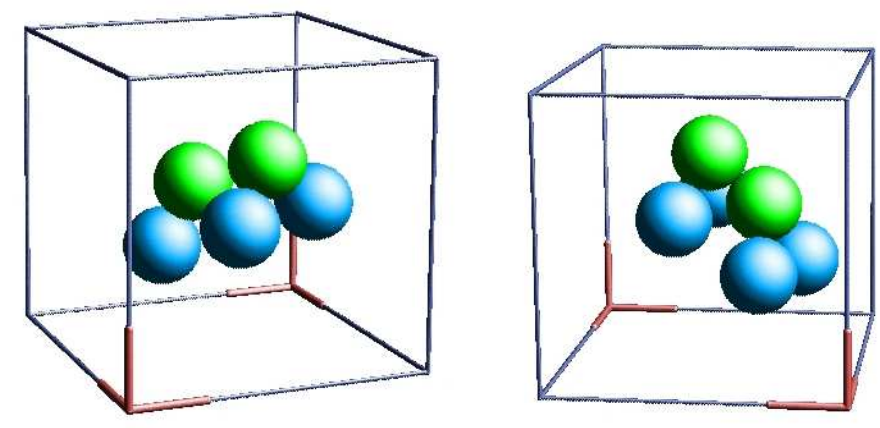

Fig. 1. Simple two particle bridge with three and four base particles

\section{Model deposits}

We have examined bridge structures in hard sphere assemblies that are generated by an established, non-sequential, restructuring algorithm, [9, 10]. This algorithm restructures a stable, hard sphere, deposit in three distinct stages. Firstly free volume is introduced homogeneously throughout the system and the particles are given small, random, displacements. Secondly the packing is compressed in a uniaxial external field using a low-temperature Monte Carlo process. Thirdly the spheres are stabilized using a steepest decent 'drop and roll' dynamics to find a local minimum of the potential energy. Crucially, during the third phase of the restructuring the spheres, although moved in sequence, are able to roll in contact with spheres that are in either stable or unstable positions; thus mutual stabilizations may arise. The final configuration has a well defined network of contacts and each sphere has a uniquely defined three point stability (In practice the final configuration may include a few 'rattlers', [3]).

Restructuring simulations are performed in a rectangular cell (a square prism) with periodic boundaries in the lateral directions and a hard, disordered base perpendicular to the compression (external field) direction. Our previous investigations, 9], have shown that, this restructuring process does not depend strongly on the simulation parameters and that, after many cycles, restructured packings have a steady state described by particular values for the structural descriptors such as the mean packing fraction and the mean coordination number. Typically the steady state mean volume fraction is in the range $\phi \sim 0.55-0.61$ and the mean coordination number is $Z \sim 5.6 \pm 0.1$. The nature of the steady state is determined by the size of the expansion phase or the 'amplitude' of the process, [9]. We have shown, 10,11, that the random packings generated in this way have many features in common with the states generated in vibrated granular media. In particular we have shown that by varying the driving amplitude systematically we can explore 'irreversible' and 'reversible' branches of a density versus driving amplitude rela- 
tionship analogous to the experimentally observed behaviour, [12]. We have used monosized spheres in order to avoid any problems with induced size segregation and a disordered base prevents ordering. In the packings we have considered here the $Q_{6}$ order parameter, e.g. [3], has a value $Q_{6} / Q_{6}^{f c c} \sim 0.05$. This small value indicates only a very limited amount of face centered cubic crystalite formation in the system $\left(Q_{6}^{f c c}\right.$ is the value of the order parameter for a face centered cubic crystal structure).

\section{Statistics of bridge structures}

We have identified clusters of mutually stabilized particles in computer generated packings of hard spheres. Each configuration includes approximately $N_{t o t}=2500$ particles and we have examined approximately 100 configurations from each of two steady states, with $\phi=0.56$ and 0.58 , of the reorganization process.
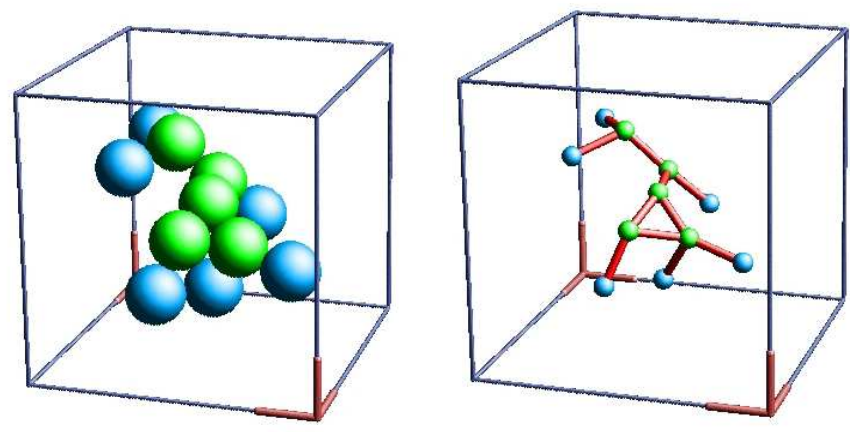

Fig. 2. A five particle bridge with six base particles and the corresponding contact network

Figure 2 illustrates a mutually stabilized cluster of five particles that is part of a large, stable, packing; this figure also shows six particles which form a base (all other particles in the packing are hidden to make the diagram). Also shown in figure 2 is the network of contacts for the particles in the bridge. This bridge is quite complex and includes a set of three particles (lower and to the right) that each have two mutual stabilizations.

Figure 3 illustrates a seven particle bridge with nine base particles. The contact network shows that although this bridge is larger than that in figure 2 it has a simpler topology because all of the mutually stabilized particles are in sequence the bridge is string-like. The right hand configuration in figure 1, with four base particles, is a string-like bridge. In practice string like bridges are common; bridges such as the one illustrated on the left hand side of figure 1 are very rare in our packings.

Each packing contains a large variety of bridge sizes and shapes. Approximately 

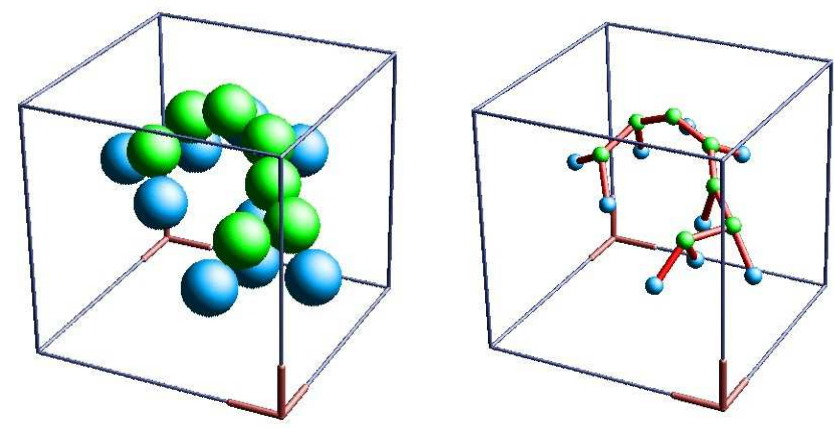

Fig. 3. A seven particle string-like bridge with nine base particles and the corresponding contact network

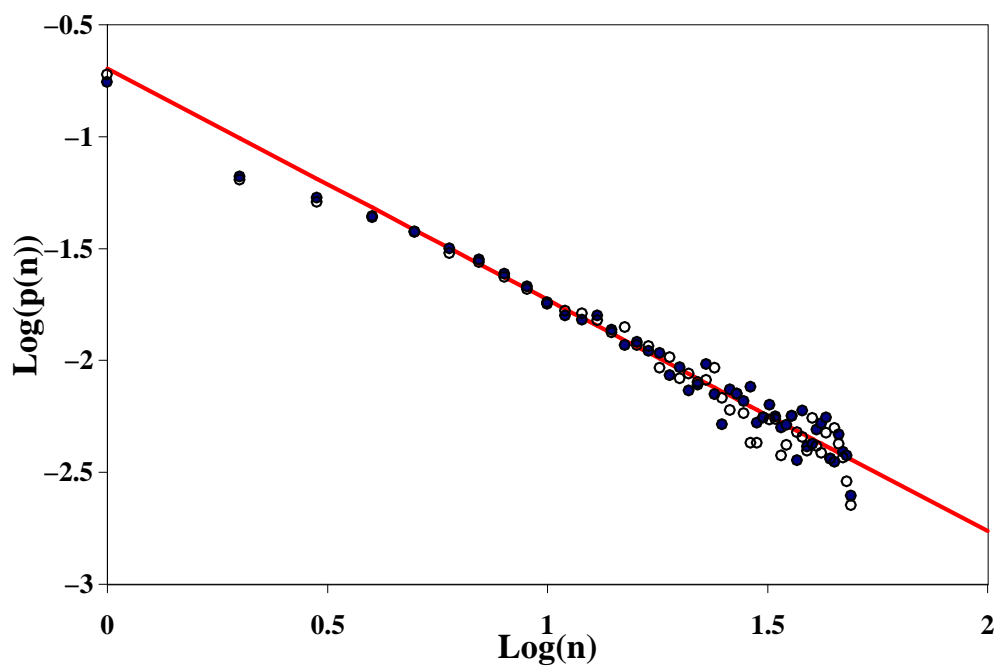

Fig. 4. The size distribution of bridges in non-sequentially reorganized hard sphere deposits; the full circles correspond to packings with $\langle\phi\rangle=0.58$, the open circles correspond to packings with $\langle\phi\rangle=0.56$ and the line is a fitted scaling $p(n) \sim n^{-\alpha}$.

80 percent of particles are in mutually stabilized locations. In figure 1 we have plotted the size distribution of the bridges as $\log (p(n))$ against $\log (n)$ where $p(n)=<n N(n) / N_{t o t}>$ and $N(n)$ is the number of bridges which contain $n$ mutually stabilized particles. Angular brackets indicate an average over configurations in the steady state. We can consider $p(n)$ as the probability that a particular particle is included in a bridge with size $n$. Over a wide range of bridge sizes the distribution function has a scaling behaviour of the form $p(n) \sim n^{-\alpha}$ with $\alpha \sim 1.0 \pm 0.03$. The bridge size distribution is not strongly dependent on the volume fraction of the packings. 


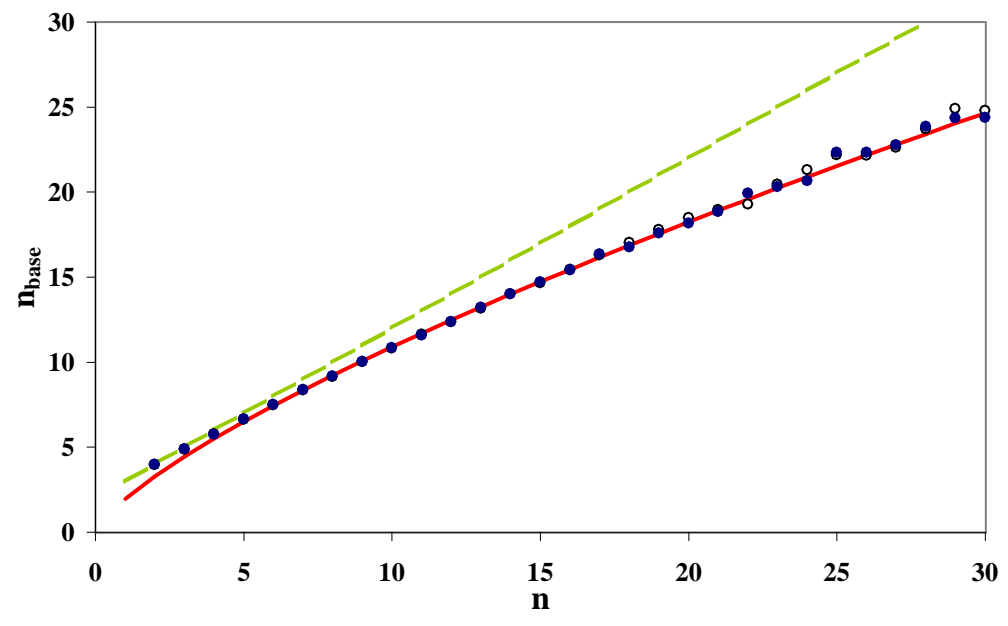

Fig. 5. The mean base size for bridges with size $n$; the dashed line indicates behaviour for stringlike bridges $n_{\text {base }}=n+2$ and the full line is a scaling fitted to the behaviour of the larger bridges.

For a particular bridge size the number of base particles, which complete the stabilization, is variable with an upper bound, $n+2$, corresponding to a string-like bridge. The mean number of base particles, $n_{\text {base }}$, is plotted as a function of the bridge size in figure 5 . There is a crossover in behaviour at $n \sim 8$; small bridges are predominantly string-like and larger bridges have more complex structures with relatively fewer base particles. Again this property is not strongly dependent on the volume fraction of the packings in the range we have considered. We did not observe any 'domes' or 'canopies' although this could be an artefact of the relatively small sizes of the deposits. For a particular bridge configuration a triangulation of the base particles can be used to construct a unique bridge axis as the mean of the triangle normals. With respect to this axis geometrical descriptors, such as the radius of gyration or the aspect ratio, also show a cross over that indicates the significance of a sub-population of string-like bridges.

A string-like bridge has uniquely defined end particles and, therefore, a well defined extension. The mean squared separation, $\left\langle r_{n}^{2}\right\rangle$, of the end particles for string-like bridges scales with the number of stabilizing bonds according to $<r_{n}^{2}>\sim(n-1)^{\gamma}$ with $\gamma=1.33$. This 'superdiffusive' behaviour is illustrated in figure 6. The population of string-like bridges we observe, in reorganized three dimensional deposits, is thus distinct from the random walk structures that have been identified as the cause of blocking at the outlet of a two dimensional hopper, [8].

Figure 7 shows the distribution function of base extensions for all bridges in packings that are part of the restructuring steady state with $\phi=0.58$. The extension, $b_{x}$, is the projection, in a plane perpendicular to the external field, of the 


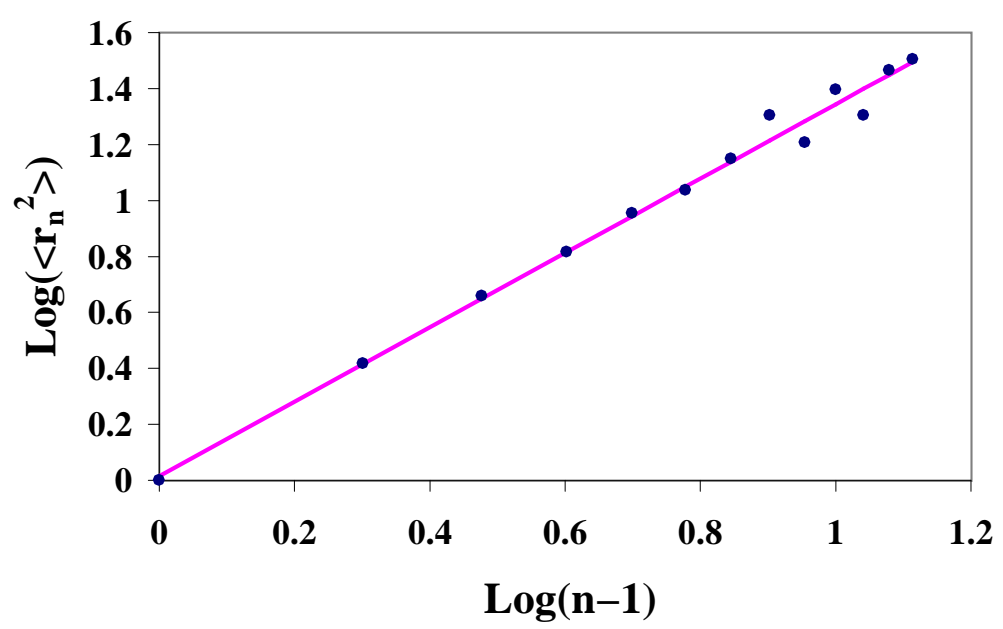

Fig. 6. The mean squared displacement, $\left\langle r_{n}^{2}\right\rangle$, for string-like bridges as a function of the number of mutually stabilizing bonds $n-1$. Bridges are part of restructured deposits with steady state volume fraction $\phi=0.58$.
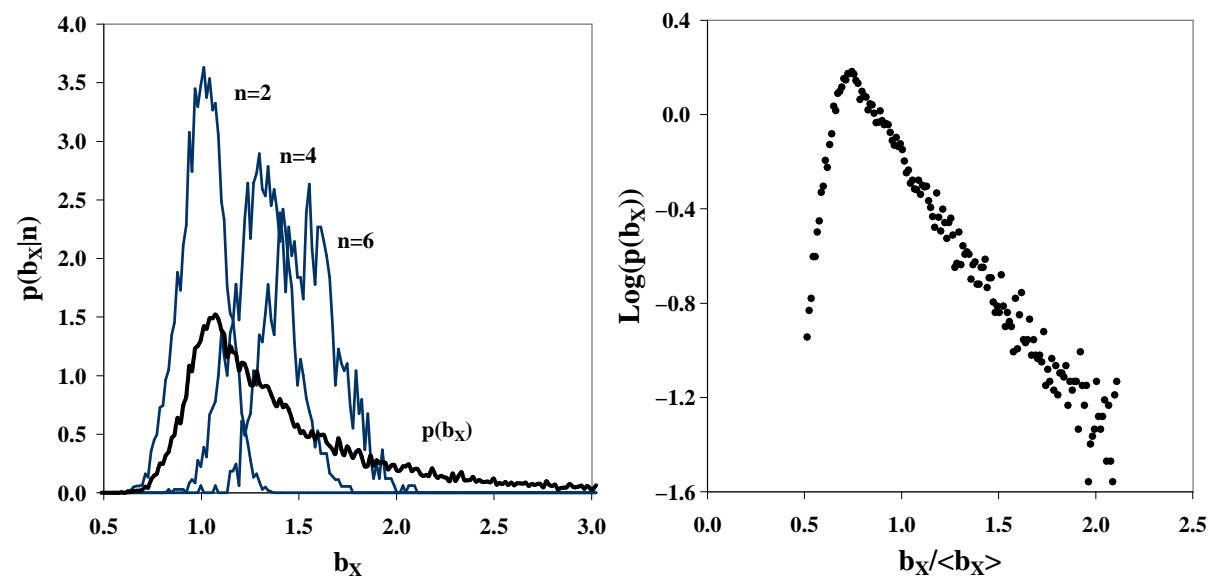

Fig. 7. The distribution of base extensions for bridges that are part of restructured deposits with steady state volume fraction $\phi=0.58$. The left hand figure also shows the distributions conditional on the bridge size, $n$, for $n=2,4,6$. The right hand figure shows the logarithm of the density as a function of the normalized variable $\left.b_{x} /<b_{x}\right\rangle$.

radius of gyration of the base particle configuration (about the bridge axis). Clearly this measure is related to the ability of a bridge to span an opening and, therefore, is an indicator of the jamming potential for a bridge. We have also shown, in figure 7. distribution functions that are conditional on the bridge size $n$ (for $n=2,4,6$ ). The conditional distributions are sharply peaked, and are bounded at finite $b_{x}$, but the total distribution has a long tail, at large extensions, reflecting the existence of 
large bridges. In the second part of figure 7 we have plotted the logarithm of the probability density against a normalized variable, $b_{x} /\left\langle b_{x}\right\rangle$, where $\left\langle b_{x}\right\rangle$ is the mean extension of bridge bases. This figure emphasizes the exponential tail of the distribution function and also shows that bridges with small base extensions are unfavoured. The absence of bridges with base extensions that are considerably smaller than the mean extension is a reflection of the angular constraints that exist in hard particle structures. Small base extensions reduce the number of possible stable configurations for bridges with fixed size $n$. The form of the distribution in figure 7 can be interpretted, clearly, in terms of a partition, $p\left(b_{x}\right)=\sum_{n} p\left(b_{x} \mid n\right) p(n)$, since the conditional probabilities have restricted ranges, reflecting hard particle volume and angular constraints, and the size distribution has a well defined scaling that reflects the particular bridge creation and anhiliation processes that are included in the restructuring. In this form it is clear that the tail of the distribution of $b_{x}$ arises from the summation and not from bridges with a particular size. It is interesting to note that the form of the normalized distribution in figure 7 is similar to the distribution of the normal forces in dense packings of hard particles, e.g. 13.

\section{Discussion}

Bridges and arches are significant elements of the mesostructure in many granular solids processing scenarios, e.g. [7, 14]. These structures, which extend beyond the scale of single particles, are strongly associated with important macroscopic properties of materials and with flow instabilities. We have shown that bridge structures are included throughout the non-sequentially reorganized deposits we have constructed. Bridges have well defined statistics and, to a first approximation, they are distributed homogeneously within the deposits. We have identified a sub-population of bridges, which have string-like configurations, that dominate for low bridge sizes. At present it is unclear whether these structures are a property of the particular reorganization scheme considered here or whether they are a fundamental feature of non-sequential reorganization in hard sphere deposition. The bridge size statistics we have presented do not depend strongly on the volume fraction of the deposits but other measures, such as the bridge orientations (which we will report elsewhere), do vary with packing density i.e. with the expansion amplitude of the reorganization process. Additionally it is clear that non-sequential structures like bridges, that become trapped in the close packed systems, frustrate local ordering in packings of monosized spheres. Thus the onset of ordering must coincide with changes in the distribution of bridges; for driving amplitudes that are smaller than those used to construct the deposits considered above we have observed the sudden onset of ordering, [15]. We have not examined correlations of the bridges in the series of packings generated by the reorganization process.

Clearly, based on an assumption that the bridges in bulk are the same as those close to an opening, the statistics of extended structures in hard particle deposits is sufficient to estimate the probability that a bridge will form a span at an outlet of a 
fixed size. In three dimensions this probability is not the same as the probability that a bridge will form a blockage or a 'jam'. However initial investigations, [16], indicate that data, analogous to the complement of the cumulative form of the distribution in figure 7 , are in qualitative agreement with observations of the jamming probability. We hope to present details of these analyses in a future report.

\section{Acknowledgements}

GCB acknowledges support from the Fundación Antorchas, and the hospitality of Prof. J. Raul. Grigera, during a visit to IFLYSIB, La Plata, Argentina where some of this work was completed. LAP acknowledges support from the International Union of Pure and Applied Biophysics during a visit to IFR, UK.

\section{References}

[1] D. J. Cumberland and R. J. Crawford, The Packing of Particles (Elsevier, Amsterdam, 1987).

[2] James G Berryman, Phys. Rev. B27, 1053 (1983).

[3] S. Torquato, T. M. Truskett, P. G. Debenedetti, Phys. Rev. Lett. 84, 2064 (2000).

[4] J. D. Bernal, Nature 183, 141 (1959).

[5] G. T. Seidler, G. Martinez, L. H. Seeley, K. H. Kim, E. A. Behne, S. Zaranek, B. D. Chapman, S. M. Heald, D. L. Brewe , Phys. Rev. E62, 8175 (2000).

[6] R. M. Nedderman, U. Tuzun, S. B. Savage, G. T. Houlsby, Chem. Eng. Sci. 37, 1597 (1982).

[7] R. L. Brown, J. C. Richards, Trans. Inst. Chem. Eng. 38, 243 (1960).

[8] Kiwing To, Pik-Yin Lai, K. K. Pak, Phys. Rev. Lett 86, 71 (2001).

[9] Anita Mehta and G. C. Barker, Phys. Rev. Lett. 67, 394 (1991).

[10] G. C. Barker and Anita Mehta, Phys. Rev. A45, 3435 (1992).

[11] G. C. Barker and Anita Mehta, Phys. Rev. E47, 184 (1993).

[12] E. R. Nowak, J. B. Knight, E. Ben-Naim, H. M. Jaeger, S. R. Nagel, Phys. Rev. E57, 1971 (1998).

[13] C. S. O'Hern, S. A. Langer, A. J. Liu, S. R. Nagel, Phys. Rev. Lett. 86, 111 (2001).

[14] P. Cooper, Bulk Solids Handling. 8, 162 (1988).

[15] Anita Mehta and G. C. Barker, J. Phys. Condensed Matter. 12, 6619 (2000).

[16] L. A. Pugnaloni, Unpublished. 